\title{
Orbital wall restoring surgery with primary orbital wall fragments in blowout fracture
}

\author{
Dong Hee Kang \\ Department of Plastic and \\ Reconstructive Surgery, Dankook \\ University Hospital, Cheonan, Korea
}

\begin{abstract}
Most orbital surgeons believe that it's difficult to restore the primary orbital wall to its previous position and that the orbital wall is so thin that cannot be firmly its primary position. Therefore, orbital wall fractures generally have been reconstructed by replacing the bony defect with a synthetic implant. Although synthetic implants have sufficient strength to maintain their shape and position in the orbital cavity, replacement surgery has some drawbacks due to the residual permanent implants. In previous studies, the author has reported an orbital wall restoring technique in which the primary orbital wall fragment was restored to its prior position through a combination of the transorbital and transantral approaches. Simple straight and curved elevators were introduced transnasally to restore the orbital wall and to maintain temporary extraorbital support in the maxillary and ethmoid sinus. A transconjunctival approach provided sufficient space for implant insertion, while the transnasal approach enabled restoration of the herniated soft tissue back into the orbit. Fracture defect was reduced by restoring the primary orbital wall fragment to its primary position, making it possible to use relatively small size implant, furthermore, extraorbital support from both sinuses decreased the incidence of implant displacement. The author could recreate a natural shape of the orbit with the patient's own orbital bone fragments with this dual approach and effectively restored the orbital volume and shape. This procedure has the advantages for retrieving the orbital contents and restoring the primary orbital wall to its prior position.
\end{abstract}

Keywords: Blow-out fracture / Enophthalmos / Orbital fracture

\section{INTRODUCTION}

Blowout fractures are the defects located deep in the bony orbit and reconstruction of these complicated areas remains as a challenge for orbital surgeons. The surgical goal in the treatment of blowout fractures is to restore ocular motility and the previous orbital shape to prevent enophthalmos [1]. The surgeon should carefully reposition the herniated orbital contents and the fractured orbital wall in their prior positions. However,

\footnotetext{
Correspondence: Dong Hee Kang

Department of Plastic and Reconstructive Surgery, Dankook University Hospital, 201 Manghyang-ro, Dongnam-gu, Cheonan 31116, Korea

E-mail: dhkcool@daum.net

Received November 30, 2019 / Revised December 13, 2019 /

Accepted December 13, 2019
}

most orbital surgeons believe that it is difficult to restore the primary orbital wall to its previous position and that the orbital wall is so thin that it cannot be fixed firmly to its primary position [2]. Therefore, orbital wall fractures generally have been reconstructed by replacing the bony defect with an autologous bone graft or synthetic implant [2,3]. Although those implants are strong enough to maintain their shape and position in the orbital cavity, replacement surgery using this method has some drawbacks [2,4]. First, a large implant is required to bridge the entire fracture defect from the medial to lateral edges of the fracture. Second, when an orbital fracture is repaired with an artificial implant without restoring the primary bone fragment, the thin flexible implant tends to be displaced into the paranasal sinus because there is no supporting structure. Third, per- 
manent non-resorbable alloplastic implants (e.g., titanium or porous polyethylene) are widely used, but can cause late complications including infection and foreign body reaction $[4,5]$.

The author has reported an orbital wall restoring technique in which the primary orbital wall fragment is restored to its prior position through a combination of the transorbital and transantral approaches, and maintaining temporary extraorbital support in the maxillary and ethmoid sinus [2-6]. This dual approach procedure combines the advantages of each approach for retrieving the orbital contents and restoring the original natural shape of the orbital wall. A transconjunctival approach provided sufficient space for implant insertion, while the transnasal approach could minimize traction injury when restoring the herniated soft tissue back into the orbit from the sinus [2-6]. In this paper, the author summarizes five articles and a recent presentations reporting an orbital wall restoration technique that applied to pure medial, floor and large inferomedial fractures as well as combined orbital wall fractures and discusses the use of a resorbable mesh plate if there is no fracture defect after this restoration technique [4,7]. Four other papers were reviewed that evaluated the clinical outcomes of the orbital wall restoration technique [8-10].

\section{MEDIAL WALL RESTORATION}

Under general anesthesia, a standard technique involving a transconjunctival incision with lateral canthotomy was used to expose the inferior orbital rim and the periosteum of the orbital rim was exposed and incised. After the nasal cavity was decongested with epinephrine pledgets, $2 \%$ lidocaine with 1:100,000 epinephrine was injected into the anterior root of the middle turbinate and a straight Freer elevator was inserted into the nose and passed gently through the ethmoidal air cells to reach the medial side of the orbital wall fracture without ethmoidectomy (Fig. 1). The prolapsed orbital contents were freed from an entrapment through an orbital approach, and the medial wall bone fragments were gently mobilized with a Freer elevator from the ethmoidal sinuses under direct vision from the transorbital approach (Fig. 2). To support the restored orbital wall, Nasopore (Polyganics B.V.) was placed in the ethmoid sinus through a transnasal approach to the medial side of the reconstructed orbit. It was absorbed several weeks later and the packing did not need to be removed [6].

\section{INFERIOR WALL RESTORATION}

A fractured orbital floor was exposed along through the subperiosteal space with a conventional transconjunctival incision and a curved Freer elevator was introduced to the maxillary sinus through the maxillary ostium (Fig. 3). The fracture fragment and herniated orbital contents were gently raised with the curved elevator from the maxillary sinus [3]. The restored orbital wall could be confirmed through the transorbital view, and an implant could be placed over the restored floor subperiosteally. To support the restored orbital floor, the balloon of a 14-16 Fr. Foley catheter (Sewoon Medical Co., LTD) was held in curved mosquito forceps, and inserted into the maxillary sinus through the maxillary ostium (Fig. 3). A ballooning volume appropriate for each patient ranging from 10 to $17 \mathrm{~mL}$ of saline mixed with contrast agent (Telebrix 30) was injected to monitor the balloon after surgery until the floor was restored to its primary position. The tube of a Foley catheter was fixed on the cheek and the balloon was drained and removed 1 week after the operation [3].
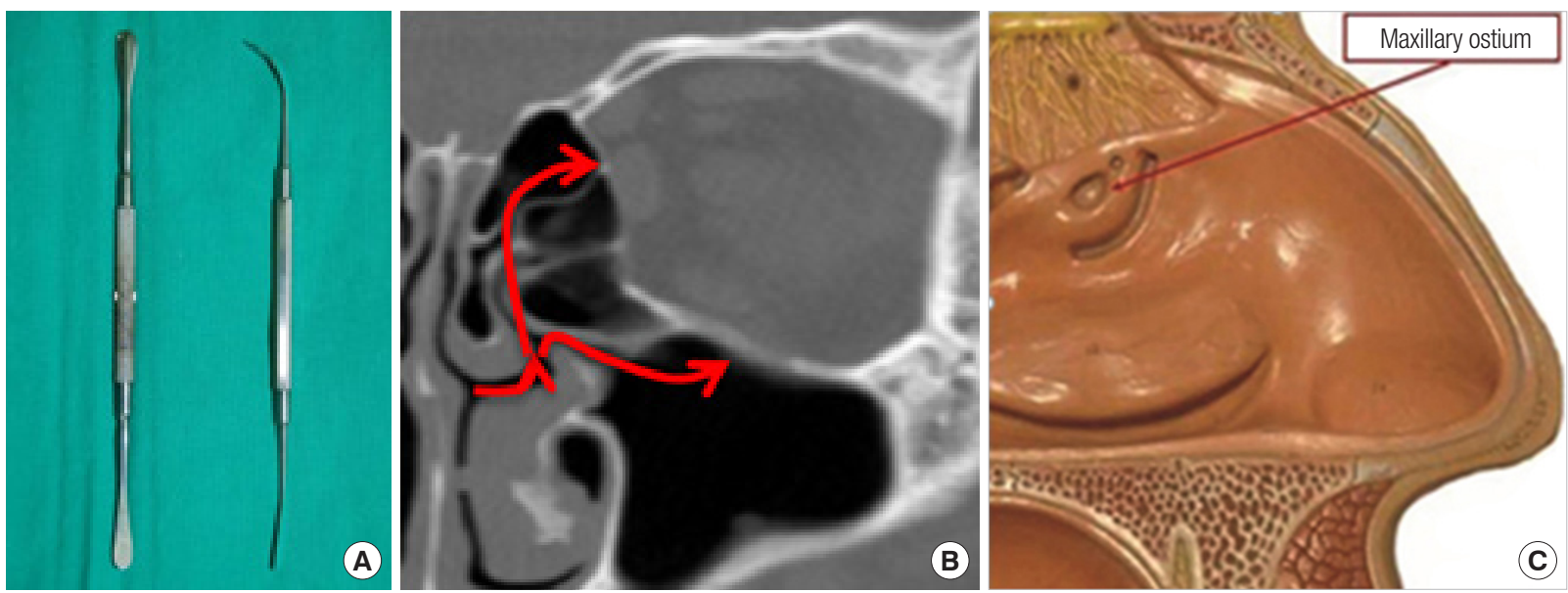

Fig. 1. (A) Simple straight and curved elevators used in transnasal restoration. (B) Transnasal approach (red arrows). (C) Maxillary ostium used for floor restoration. Adapted from Lim et al. Ann Plast Surg 2015;75:522-5, with permission [3]. 


\section{LARGE INFERIOR MEDIAL WALL RESTORATION}

Compared to isolated medial or inferior blowout fractures, the surgical treatment of a large inferomedial wall blowout fracture presents a particular challenge for the orbital surgeon. Infero- medial blowout fractures are accompanied by a wider range of fractures than other isolated floor or medial orbital wall fractures, and they show a high rate of complications such as enophthalmos, diplopia and severe volume expansion of the orbital cavity [1,5]. Several techniques using a variety of implants have been described to reconstruct wide inferomedial orbital
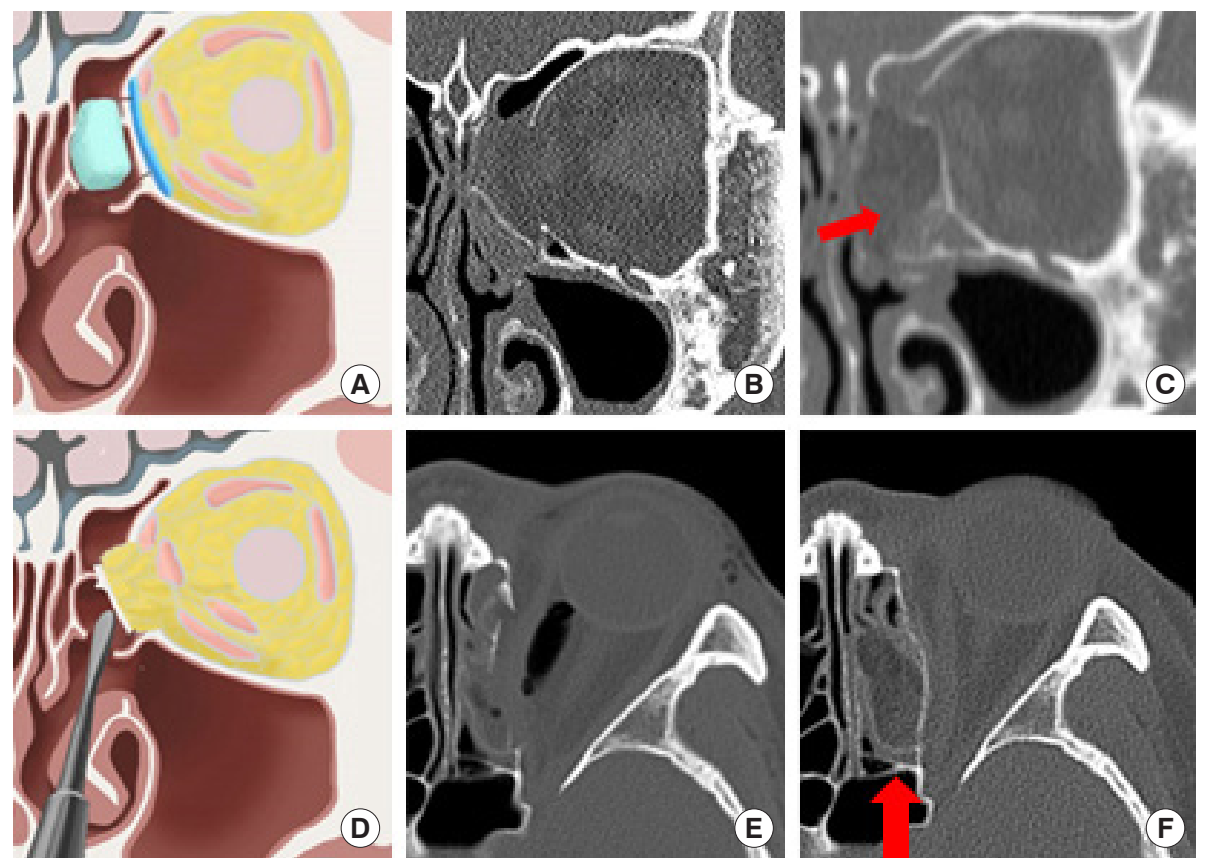

Fig. 2. (A, D) Medial wall was restored with straight elevator from ethmoid sinus and supported with Nasopore. (B, E) Preoperative CT. (C, F) Postoperative CT. Nasopore (red arrow). CT, computed tomography.
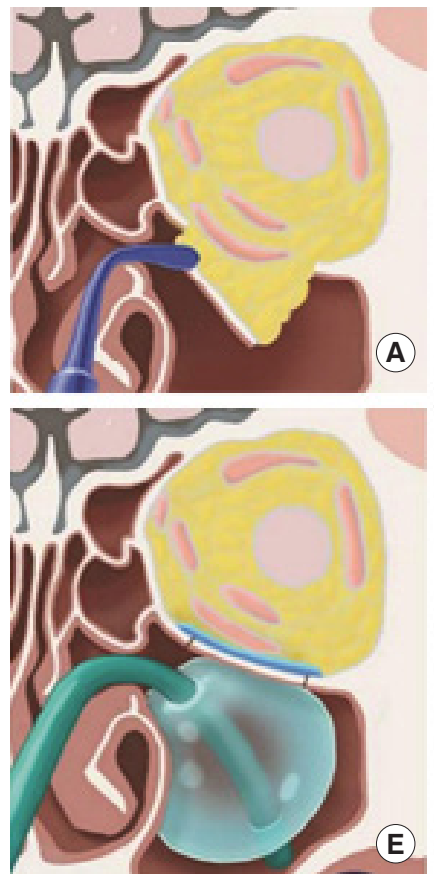
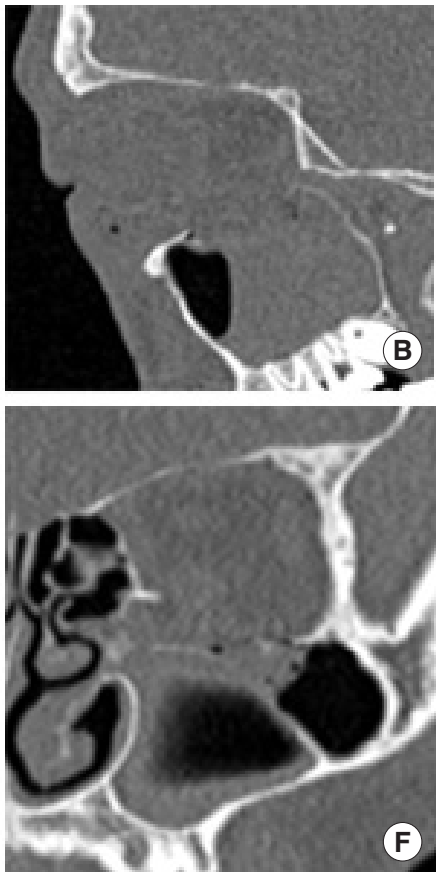
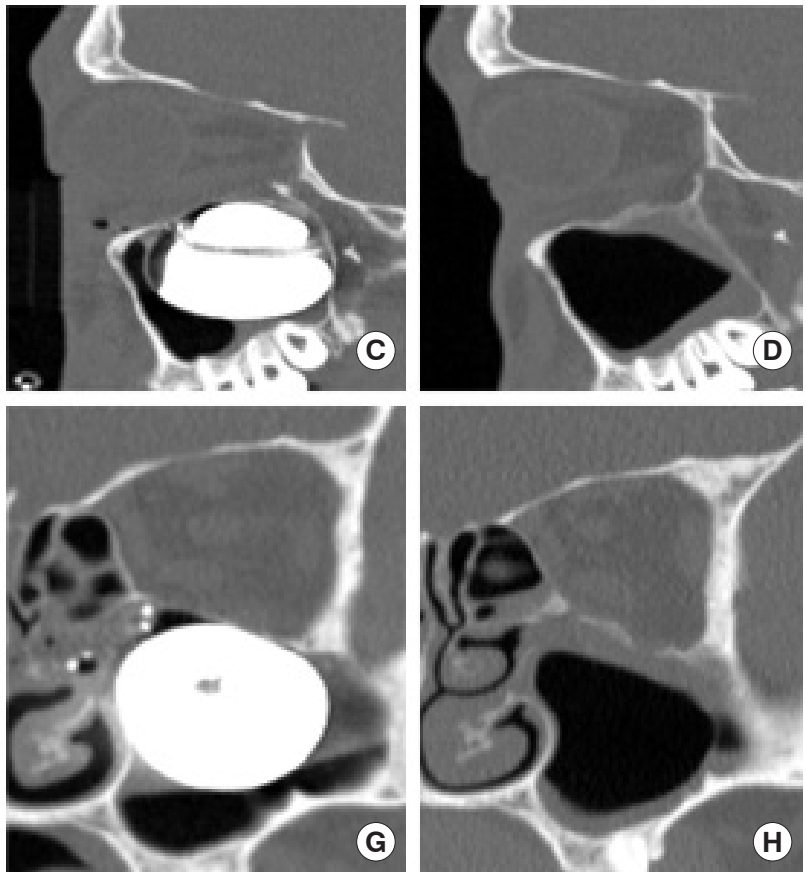

Fig. 3. (A, E) The restored floor was temporary supported by balloon. (B, F) Preoperative CT. (C, G) Postoperative CT. (D, H) Six months follow-up CT. CT, computed tomography. Ann Plast Surg 2015;75:522-5, with permission [3]. 

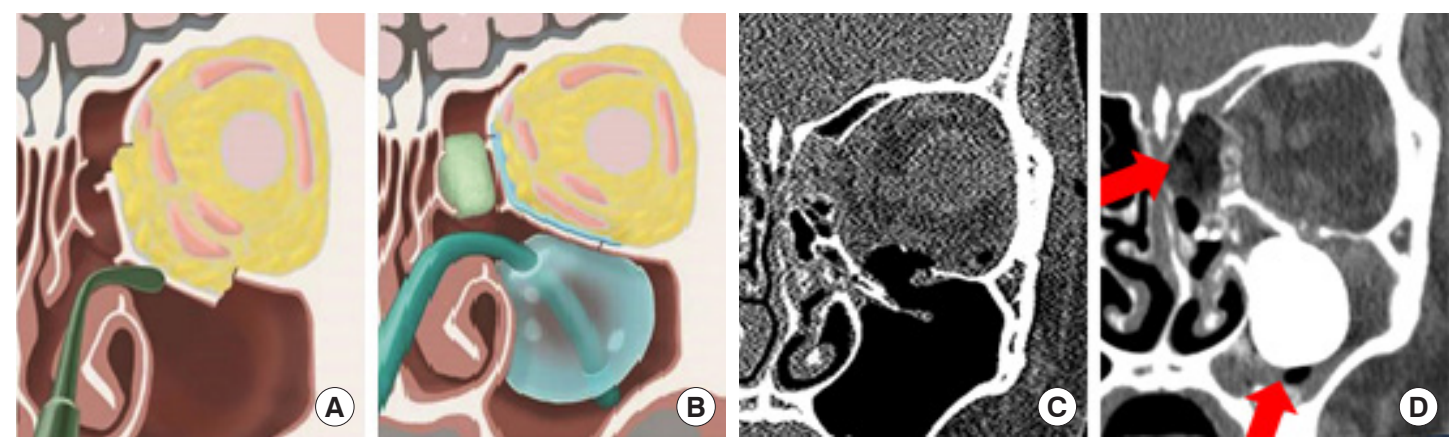

Fig. 4. (A, B) Transnasal restoration of the inferomedial orbital wall fracture. Restored orbital wall was supported with resorbing form and Foley balloon from ethmoid and maxillary sinus. (C) Preoperative CT. (D) Postoperative CT: resorbing form and Foley balloon in ethmoid and maxillary sinus (red arrows). CT, computed tomography. Adapted from Lim et al. J Craniofac Surg 2015;26:e761-5, with permission [5].

wall fractures, but those methods always require large implants to bridge the entire fracture defect, from the medial to the lateral margin of the fracture [5]. However, it is difficult to recreate the natural shape of the orbit with large implants and to maintain those large implants in their proper position due to the absence of the supporting structures in inferomedial wall fractures [5]. These large implants occasionally produce unfavorable outcomes, such as implant displacement and reherniation, because of the lack of a suitable supporting structure. Furthermore, the inferomedial strut is an extraorbital supporting structure located at the ethmoid-maxillary junction that is frequently compromised in large inferomedial orbital fractures $[4,5]$. The surgical restoration of this structure always requires an extraorbital approach since transorbital restoration of the inferomedial strut is impossible. In a case of inferomedial wall fracture, restoration was achieved by using both a straight and curved Freer elevator consecutively (Fig. 4). Nasopore was inserted into the ethmoid sinus to provide support from the medial side of the orbit. A Foley catheter balloon was inserted through the maxillary ostium using a curved mosquito clamp and provided support from the maxillary sinus [5].

\section{ORBITAL WALL RESTORATION WITH A RESORBABLE MESH PLATE}

Recently, resorbable mesh plates have been increasingly used as implants in orbital wall reconstruction. Ideal resorbable orbital mesh plates should retain their mechanical strength over 1 to 2 years, allowing for adequate fibrous tissue formation in the bony orbital wall defect, and then be degraded and absorbed completely to minimize the risk of a foreign body reaction [4]. The load bearing strength of mesh plates is adequate for use in isolated floor and medial wall fractures with an intact bony buttress, but they are not recommended for use in large orbital wall fractures that need load bearing support. Implants placed in large orbital wall fractures have been reported to show buckling and sagging due to the loss of their mechanical properties [11], and there is some risk of late enophthalmos after total absorption; thus, the long-term results are questionable [4].

The bone defect of the orbital wall became smaller as the fractured orbital wall was restored to prior position with patient's own orbital wall fragment. The temporary extraorbital support was expected to reduce the load applied on the orbital implants and to decrease the risk of postoperative buckling and sagging of resorbable implants [4]. Therefore, the use of resorbable implants was expected to be reasonable in the author's orbital wall restoring technique to avoid the late complications of residual permanent implant.

\section{ORBITAL WALL RESTORATION OF COMBINED ORBITAL WALL FRACTURE}

The orbital wall restoration method was thought to be difficult to use for complex orbital wall fractures, since the sharp screw tip used for buttress fixation increases the risk of balloon rupture. However, we could avoid the balloon rupture by fixing the screw at the margin of the buttress [7], which yield successful outcomes in complex orbital wall fracture (Fig. 5).

\section{CAN ENOPHTHALMOS BE A SURGICAL INDICATION FOR BLOWOUT FRACTURE?}

Traditional indications for the open reduction of orbital fractures have included the presence of symptoms such as limitation of extraocular muscle motion, radiological evidence of an extensive fracture (fracture size, $>2 \mathrm{~cm}^{2}$ ), and enophthalmos produced by an orbital volume expansion [1]. However, enophthalmos may not appear immediately after trauma due to trau- 

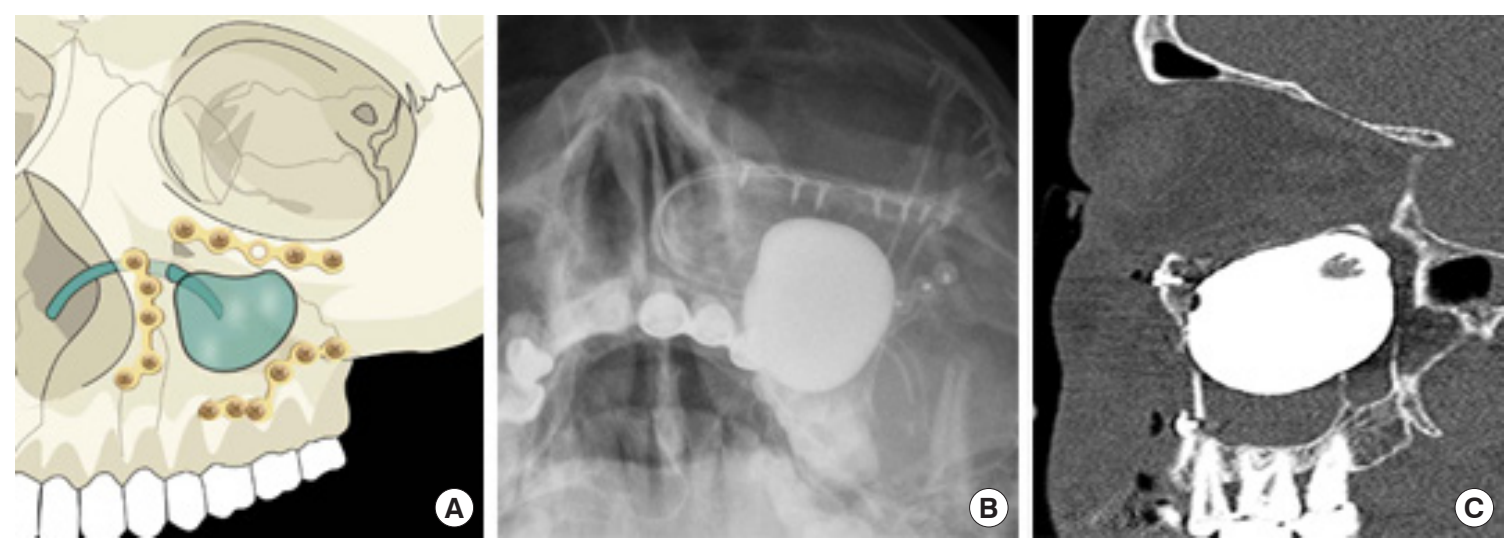

Fig. 5. (A) By fixing the screw at the edge of the buttress, it was possible to avoid the balloon rupture in combined orbital wall fracture. (B) Postoperative X-ray. (C) Postoperative computed tomography.

matic edema; furthermore, it is difficult to detect enophthalmos even before surgery due to residual swelling $[1,8]$. The periorbital swelling caused by trauma makes it difficult to measure the exact amount of enophthalmos, and measurements of the final stable degree of enophthalmos are frequently delayed by several months after the resolution of periorbital swelling $[9,10]$. Therefore, preoperative measurements cannot be used as a reliable guideline for the orbital surgeon, but preoperative prediction of the eventual final extent of enophthalmos is essential in order to determine the need for surgery $[9,10]$.

\section{PREDICTION OF LATE ENOPHTHALMOS WITH COMPUTED TOMOGRAPHY MEASUREMENTS}

In cases where patients do not have any limitations of ocular movement, the ophthalmic surgeons need to determine whether to perform an operation by evaluating preoperative computed tomography (CT) measurements. More specifically, they should able to predict the eventual final degree of enophthalmos based on the information from the preoperative CT measurements $[9,10]$. Several attempts have been made to predict the extent of late enophthalmos using preoperative orbital volume measurements with orbital CT, but volume measurements of the orbit have not previously been used for surgical guidelines. Preoperative CT measurements provide valuable data that can be used to plan appropriate treatment for blowout fracture with the advancement of the technology. Therefore, orbital surgeons should likewise be able to predict the final extent on the basis of preoperative CT measurements $[9,10]$.

It has long been known that enophthalmos is associated with volume expansion and other factors, such as fracture area and soft tissue atrophy. Although measurements of the final degree

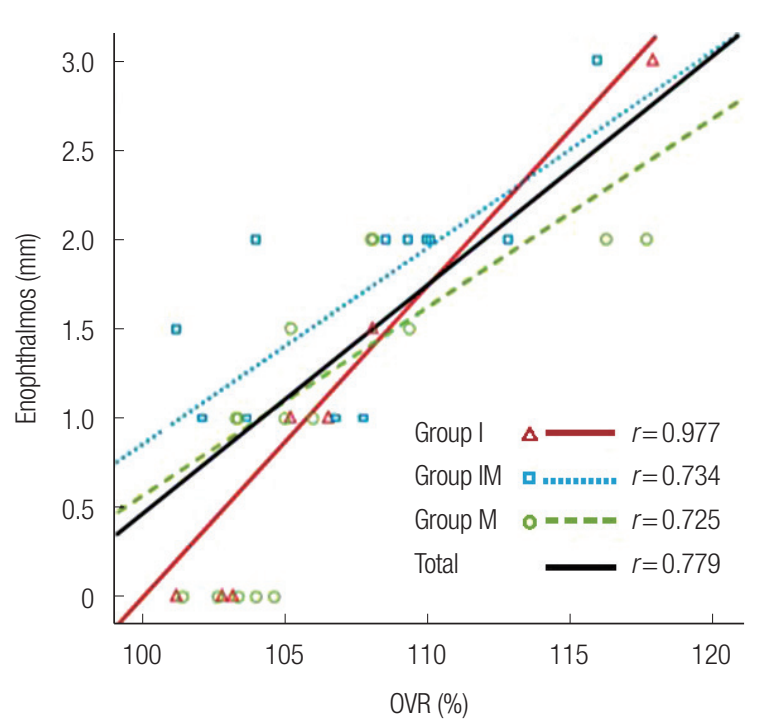

Fig. 6. Correlations between the orbital volume ratio (OVR) and enophthalmos in unoperated blowout fractures. Group I, inferior wall fracture group; group IM, inferomedial wall fracture group; group $M$, medial wall fracture group. Reprinted from Choi et al. Archives Plast Surg 2016;43:518-22 [9].

of enophthalmos are usually delayed by several months until the resolution of periorbital edema and the progression of soft tissue atrophy, numerous studies have used preoperative CT measurements to predict enophthalmos $[9,10]$. The author reported the correlations of the orbital volume ratio (OVR) (Fig. 6) and orbital fracture ratio (OFA) with late enophthalmos in unoperated blowout fractures. Both OVR $(r=0.777)$ and OFA $(r=0.799)$ showed significant correlations with enophthalmos, therefore, OVR and OFA are quantitative values that could be used to predict late enophthalmos. OVR showed a closer correlation with enophthalmos than OFA, but there was no statistically significant difference between the two correlations $[9,10]$. According to the correlations graphs, enophthalmos of $2 \mathrm{~mm}$ could be expected with an OVR of $112.28 \%$ and an OFA of 2.63 

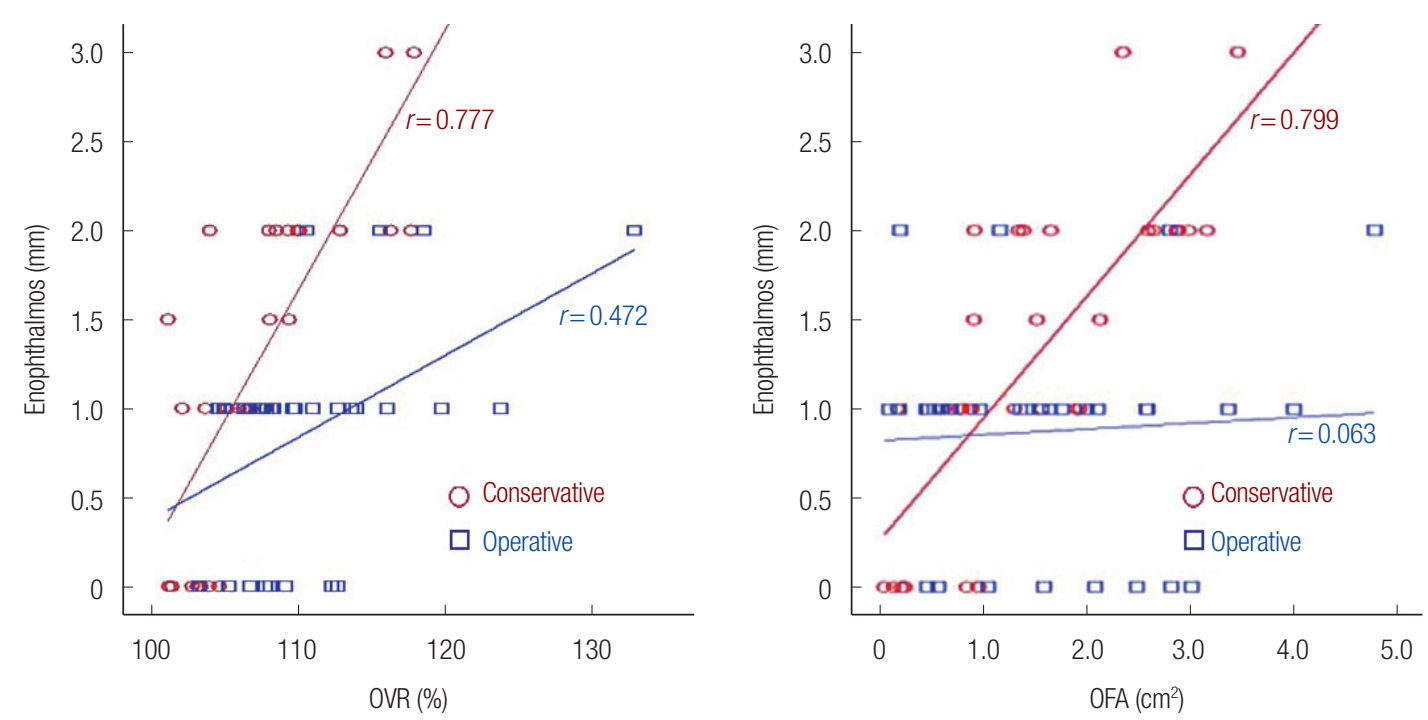

Fig. 7. Correlations between the orbital volume ratio (OVR), orbital fracture area (OFA) and the degree of enophthalmos. Enophthalmos of 2 $\mathrm{mm}$ could be expected with an OVR of $112.28 \%$ and an OFA of $2.63 \mathrm{~cm}^{2}$. Reprinted from Choi SH. J Craniofac Surg 2017;28:1717-20, with permission [10].

$\mathrm{cm}^{2}$. Based on a previous study, a surgical intervention was recommended in cases with an OVR of $112.28 \%$ or higher and an OFA above $2.63 \mathrm{~cm}^{2}$ because those thresholds were associated with $2 \mathrm{~mm}$ of enophthalmos, which was considered cosmetically significant (Fig. 7) [10].

\section{DISCUSSION}

The author previously reported an orbital wall restoring technique in which the orbital floor was restored to its prior position through the transnasal approach and temporary extraorbital support was maintained with a balloon in the maxillary sinus [2-6]. This procedure significantly decreased the extent of the orbital bone defect and increased bony continuity due to the anatomical restoration of the orbital wall and there is little risk of further volume change, since the primary orbital fragments healed in their original position. Furthermore, extraorbital ballooning temporarily provided counter-support to reduce the load applied on the implant [2-6].

Since the use of a balloon to provide support for the restored orbital floor was first reported by Johnson in 1944 [12], many authors have introduced the techniques that allows simple and rapid restoration of the orbital floor. The Foley balloon catheter is a durable, flexible and adjustable implant that provides support to the restored orbital floor from the maxillary sinus, and it can be readily removed in the outpatient settings. We passed a curved Freer elevator through the maxillary ostium, it should be kept in mind, however, that a working knowledge of the complex anatomy of the nasal cavity is required to insert the in- struments through the maxillary ostium successfully without causing inadvertent trauma to the surrounding structures [2-6]. Although the location of the maxillary ostium varies, any angled instrument can easily pass through the opening to enter the maxillary sinus from the nasal cavity. Nasopore is an absorbable packing sponge that provides sufficient support from the ethmoid sinus. It was absorbed several weeks later and we did not need to remove the packing, which decreased the risk of infection [3].

The bone defect of the orbital wall became smaller as the fractured orbital wall was restored to its prior position, therefore, the use of resorbable implants in the author's orbital wall restoring technique was expected to be reasonable. In addition, the temporary extraorbital support was expected to reduce the load applied on the orbital implants and to reduce the postoperative buckling and sagging of resorbable implants in orbital wall restoration surgery [2-4].

The ideal implant should be biocompatible, sterilizable, easy to manipulate, and capable of reproducing the original orbit shape [4]. Currently, numerous alloplastic implants are produced and are commonly used to reconstruct orbital wall fractures. However, these non-resorbable permanent alloplastic implants (e.g., titanium or porous polyethylene) have late complications such as infection, foreign body reaction, displacement, and protrusion [4]. Titanium mesh may lead to the adherence of orbital structures, resulting in postoperative extraocular movement restriction or eyelid retraction [4]. Porous polyethylene remains as a foreign body, which may cause delayed-onset inflammation, despite its biocompatibility [1]. In contrast, the 
resorbable mesh plates are largely free from these disadvantages. A resorbable mesh plate retains its mechanical strength for 1 to 2 years, allowing for adequate fibrous tissue formation in the bony orbital wall defect, after which it is completely degraded and absorbed, minimizing the risk of foreign body reaction [4]. Therefore, this technique is useful for isolated floor or medial wall fractures with an intact bony buttress or a minimal fracture site [4]. However, these implants can undergo sagging or buckling due to an untimely loss of mechanical strength in large fractures [11], and they show a late enophthalmos rate of $5 \%$ to $16 \%$ [12]. The use of absorbable implants is a safe way to reduce implant deformation and complications from residual permanent implants in orbital wall restoration surgery [2-6].

In conclusion, using this orbital wall restoration technique, the author was able to recreate a natural shape of the orbit with the patient's own orbital bone fragment and effectively restored the orbital volume and shape. Fracture defect were reduced by restoring the primary orbital wall fragment making it possible to use relatively small size implant in blowout fracture surgery, furthermore, the extraorbital support provided by this technique reduced the incidence of implant displacement [2-6].

\section{NOTES}

\section{Conflict of interest}

No potential conflict of interest relevant to this article was reported.

\section{ORCID}

Dong Hee Kang https://orcid.org/0000-0002-6673-9655

\section{REFERENCES}

1. Oh SA, Aum JH, Kang DH, Gu JH. Change of the orbital volume ratio in pure blow-out fractures depending on fracture lo- cation. J Craniofac Surg 2013;24:1083-7.

2. Lim NK, Kang DH, Oh SA, Gu JH. Orbital wall restoring surgery in pure blowout fractures. Arch Plast Surg 2014;41:68692.

3. Lim NK, Kang DH, Oh SA, Gu JH. Orbital floor restoration using the transnasal balloon technique for inferior orbital wall fracture. Ann Plast Surg 2015;75:522-5.

4. Joo JD, Kang DH, Kim HS. Orbital wall restoring surgery with resorbable mesh plate. Arch Craniofac Surg 2018;19:264-9.

5. Lim NK, Kang DH, Oh SA, Gu JH. Orbital wall restoring surgery for inferomedial blowout fracture. J Craniofac Surg 2015; 26:e761-5.

6. Choi WK, Kang DH, Oh SA. Anatomical reconstruction of the medial orbital wall fracture. Arch Craniofac Surg 2012;13:2935.

7. Jung JS, Lim NK, Gu JH, Kang DH. Orbital wall restoring surgery in combined orbital wall fracture. Presented at the 22th Scientific Meeting of Korean Cleft Palate-Craniofacial Association; 2019 Nov 8-10; Seoul, Korea.

8. Jeon HB, Kang DH, Oh SA, Gu JH. Comparative study of naugle and hertel exophthalmometry in orbitozygomatic fracture. J Craniofac Surg 2016;27:142-4.

9. Choi SH, Kang DH, Gu JH. The correlation between the orbital volume ratio and enophthalmos in unoperated blowout fractures. Arch Plast Surg 2016;43:518-22.

10. Choi SH, Kang DH. Prediction of late enophthalmos using preoperative orbital volume and fracture area measurements in blowout fracture. J Craniofac Surg 2017;28:1717-20.

11. Ramesh S, Hubschman S, Goldberg R. Resorbable implants for orbital fractures: a systematic review. Ann Plast Surg 2018;81: 372-9.

12. Miki T, Wada J, Haraoka J, Inaba I. Endoscopic transmaxillary reduction and balloon technique for blowout fractures of the orbital floor. Minim Invasive Neurosurg 2004;47:359-64. 